\title{
Effect of the Dispersion State on the Dielectric Properties in High Energy Density Polymer-Based Nanocomposites
}

\author{
Nicolas Uguen ${ }^{1, *}$, Lise trouillet-Fonti ${ }^{2}$, Rabih Al Orabi ${ }^{2}$, Paul Sotta ${ }^{1}$ \\ IEEE Conference Publishing \\ ${ }^{1}$ Laboratoire Polymères et Matériaux Avancés, UMR 5268, CNRS/Solvay, 87 avenue des Frères Perret, 69192 Saint-Fons, \\ France \\ ${ }^{2}$ Polymer Materials Design and Development department (PM2D), Solvay RICL, 87 avenue des Frères Perret, 69192 Saint-
}

Fons, France

\begin{abstract}
Polymer composites with PVdF-HFP matrix and BaTiO3 nanoparticles were prepared with different dispersion states to evaluate the impact of the dispersion state of the nanoparticles on dielectric properties. We observe that the dispersion state has two effects: first, the overall dielectric permittivity is a little higher in aggregated systems than in welldispersed systems; Second, conductivity, together with a capacitive contribution, increase at low frequencies in aggregated systems as compared to well-dispersed systems. We propose that polarization couplings may enhance the effective permittivity in aggregated systems, and that Maxwell-Wagner-Sillars polarization may also contribute at low frequencies due to local percolation of the particles.
\end{abstract}

\section{INTRODUCTION}

Composites made of an electrically insulating polymer and inorganic particles may potentially offer a good compromise of properties. Adding high- $\varepsilon$ fillers increases the composite permittivity, which may lead to an increase of the energy density. However, the polarization of high- $\varepsilon$ fillers under electric field leads to the concentration of the electric field in the matrix, which generally result in the decrease of the electric strength that may reduce the energy density. Thus, to get the best compromise between permittivity, electrical losses and electrical strength, adding high- $\varepsilon$ fillers in a polymer matrix in order to increase its energy density has to be carefully considered.

During the past decades, dielectric properties of nanocomposites consisting of high- $\varepsilon$ fillers embedded in a polymer matrix have been widely studied [1], [2]. The impact of the filler permittivity, shape and orientation on the effective dielectric properties of the material has been the subject of many experimental and computational works. It is nowadays well established that it is closely related to local electric field distortion due to particles polarization. The impact of polymer-filler interfaces onto the dielectric properties of the composite has also been studied [1], [4]. The effect of the particle size is related to the drastic increase of interfacial area in nanocomposites [3]. Due to the presence of an electrical double layer at the particle vicinity, preferential charge conduction may occur at interfaces, that drastically deteriorates the electrical insulating properties of the composite if the interfaces (or interphases) percolate [3]. Interfaces may also be associated to physical disorder and chemical defects (ions, impurities...) that increase the probability of failure of the material under high electric field [5], [6]. While there is general agreement on the importance of interfacial properties for controlling dielectric properties of polymer composites, a general understanding is still missing.

More recently, the electronic structure of the composites has been increasingly taken into account when designing dielectric materials [5], [7]-[9]. Nevertheless, as well as for the impact of the interfaces, a general understanding is still missing in this matter.

When studying the effects of the interfaces and electronic structures of the components on dielectric properties and charge dynamics in polymer-based composites, a key issue is to maintain all structural parameters such as filler size, shape, orientation and dispersion state constant, as these features may have strong impact as well. In this contribution, we tune the dispersion state of $\mathrm{BaTiO} 3 / \mathrm{PVdF}-\mathrm{HFP}$ composites in order to provide insight into the relationship between the dispersion state and the dielectric properties of these composites.

\section{MATERIALS AND METHODS}

\section{A. Materials and Sample Preparation}

Polymer-based composite films were prepared by solvent casting at different volume contents $(10,5,2.5$ and 1 vol.\%) of $\mathrm{BaTiO}_{3}$ nanoparticles (Sigma Aldrich, average size about 50 $\mathrm{nm})$. In order to obtain samples with two different particle dispersion states, two different protocols were used. Firstly, in both protocols, the BaTiO3 powders are sonicated in Methyl Ethyl Ketone (MEK) for 1h. Separately, the poly(vinylidene fluoride-co-hexafluoropropane) (PVdF-HFP) (Solef 21510 from Solvay) powder is dissolved into MEK. The polymer solution is filtered through a $5 \mu \mathrm{m}$ pore Teflon membrane filter before mixing with the $\mathrm{BaTiO}_{3}$ dispersion. The ternary system is then stirred for a few minutes and spit into two vials: (1) In protocol A ("Aggregated"), the ternary solution is directly casted on a glass substrate with a Doctor Blade to obtain $20 \mu \mathrm{m}$ thick films; (2) In protocol S ("Sonicated"), the ternary system is sonicated in order to better disperse the particles into the matrix and then casted in the same conditions. Two neat PVdF-HFP films were prepared in the same conditions A and $\mathrm{S}$ as reference samples. All films are finally dried at $60^{\circ} \mathrm{C}$ under vacuum for $36 \mathrm{~h}$ and conditioned $48 \mathrm{~h}$ under air at $50 \%$ 
humidity (RH50) and $23^{\circ} \mathrm{C}$ before being characterized. The samples will be denoted by $\mathrm{Y} \_\mathrm{X}$, where $\mathrm{X}$ is the volume fraction of $\mathrm{BaTiO} 3$ and $\mathrm{Y}$ the protocol A or S. Sample compositions are reported in TABLE I.

TABLE I

Composition Of The Prepared Samples

\begin{tabular}{|c|c|c|}
\hline & \multicolumn{2}{|c|}{ Sample Names } \\
\hline $\mathrm{BaTiO}_{3}$ content $($ vol. $\%)$ & Protocol A & Protocol S \\
\hline 10 & A_10 & S_10 \\
\hline 5 & A_5 & S_5 \\
\hline 2.5 & A_2.5 & S_2.5 \\
\hline 1 & A_1 & S_1 \\
\hline 0 & A_0 & S_0 \\
\hline
\end{tabular}

\section{B. Characterization Technics}

The dispersion states of the samples were characterized by Scanning Electron Microscopy (SEM) analysis of the crosssection of the samples obtained by cryo-fracturing the films in liquid nitrogen. To quantify dispersion states, the SEM pictures were binarized and analyzed in order to extract distributions of particle/agglomerate areas, then expressed in terms of the diameters of disks of equivalent surfaces.

Dielectric spectra were recorded with a Solartron Analytical XM MTS from 0.1 to $10^{6} \mathrm{~Hz}$ at $0.1 \mathrm{~V}$. All data were recorded at $\mathrm{RH} 50$ and $23^{\circ} \mathrm{C}$.

\section{RESULTS AND DISCUSSION}

\section{A. Dispersion State}

Representative SEM images of all systems are shown in Fig.1. Size distributions obtained from image analysis are shown in Fig.2. The SEM images of samples elaborated with protocol A (Figure 1.a-d) show the presence of particle agglomerates randomly distributed in the PVdF-HFP matrix. Conversely, SEM images of samples obtained with protocol S (Figure 1.e-h) show well dispersed nanoparticles and no large agglomerates. Figure 2 shows the distributions of equivalent diameters for both sets of sample. The dispersion states are clearly different. Samples from protocol S show particle size distributions centered around $75 \mathrm{~nm}$ whereas samples from protocol A exhibit aggregates of 300-600 $\mathrm{nm}$. Therefore, the two different protocols used to prepare the nanocomposite films lead to clearly distinct dispersion states. Moreover, the particles/agglomerate size distributions do not seem to strongly depend on the particle amount.

\section{B. Dielectric spectroscopy}

Dielectric spectra were recorded as a function of the frequency for all systems, obtained from both protocols A and $S$. The relative dielectric constant $\varepsilon_{\mathrm{r}}$ ' and dielectric loss factor $\tan (\delta)$ are shown in Figure 3 for all samples.

First, the dielectric constant $\varepsilon^{\prime}(\omega)$ and loss factor $\tan (\delta)$ of neat PVdF-HFP samples A_0 and S_0 are similar, which proves that the second sonication step applied to the polymer does not impact these properties.

In Figure 3.a, $\varepsilon_{\mathrm{r}}$ ' decreases as the frequency increases for all samples, indicating a decrease in mobility of polar groups or ions in the materials as frequency increases. As expected, the

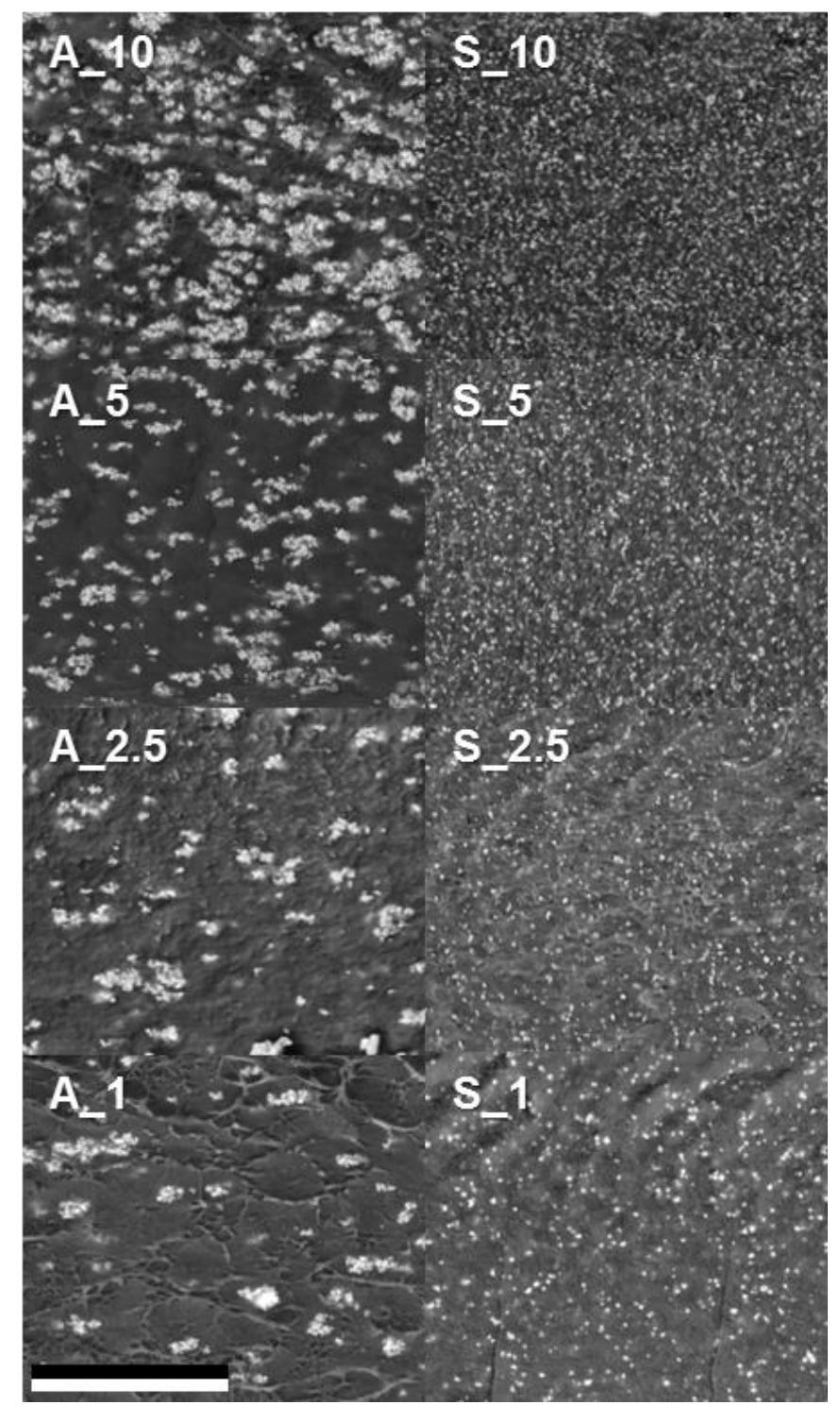

Figure 1: SEM images of the different Samples. Scale bar of $5 \mu \mathrm{m}$

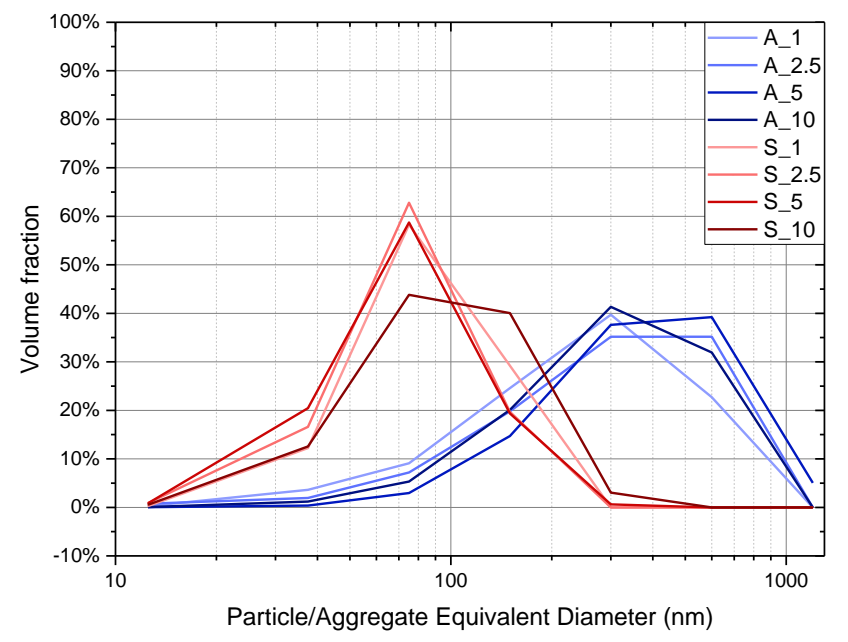

Figure 2: Size distribution function. Sample from protocol A are in blue and samples from protocol $\mathrm{S}$ are in red. 

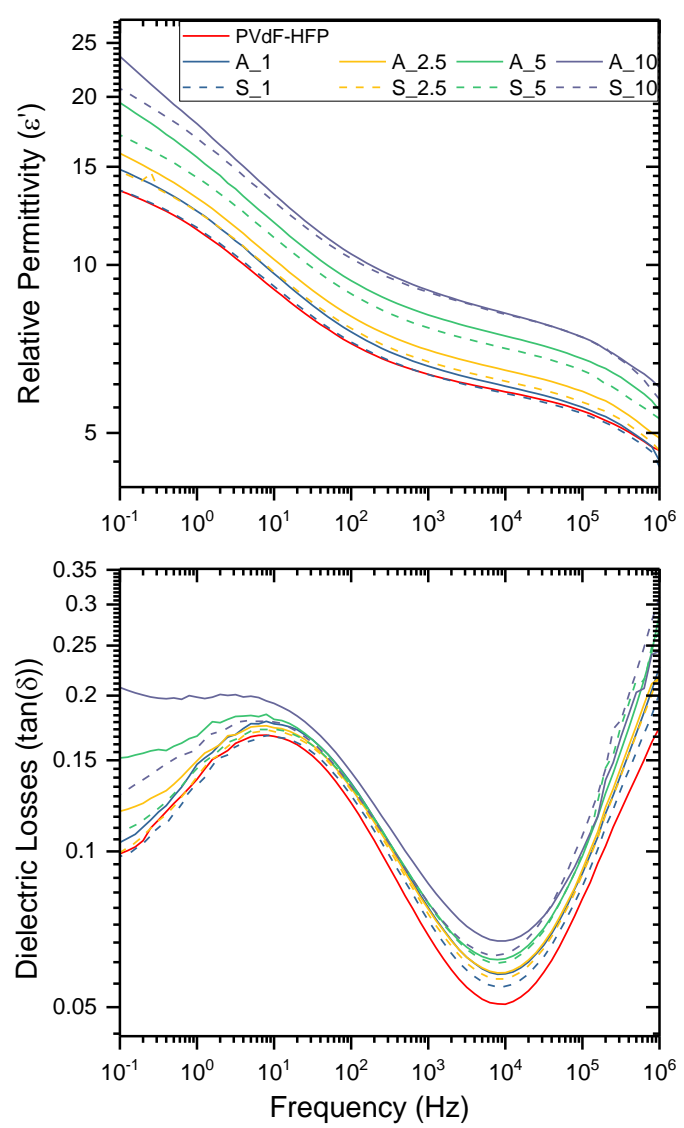

Figure 3 - Dielectric spectra of PVdF-HFP composites at $0,1,2.5,5$ and 10 vol.\% in BaTiO3 fabricated by A and S protocols (respectively full lines and dash lines). a) Permittivity and b) dielectric $\operatorname{losses} \tan (\delta)$ as a function of the frequency.

dielectric constant increases with the $\mathrm{BaTiO} 3$ volume fraction for both protocols. All composites show dielectric functions with a similar shape in logarithmic scale. Indeed, $\mathrm{BaTiO}_{3}$ has a high dielectric constant induced by ionic motion into the crystal lattice which does not depend on frequency at room temperature in the frequency range considered here [10]. Thus, on incorporating $\mathrm{BaTiO}_{3}$ particles into a PVdF-HFP matrix, the real part of the permittivity should simply be multiplied by a constant factor depending on the amount and perhaps on microstructural features of $\mathrm{BaTiO}_{3}$. This statement finds its limit at low frequency, where the rise of the dielectric loss factor brings a capacitive part leading to an increase of the effective permittivity of the composite.

Concerning the dielectric loss factor shown in Figure 3.b, the relaxation peak associated to the alpha transition of PVdFHFP is partly visible at high frequency. The peak around 10 $\mathrm{Hz}$ is related to Maxwell-Wagner-Sillars (MWS) polarization due to higher ionic conductivity in the amorphous phase of PVdF-HFP [11]. At lower frequencies, the increased $\tan (\delta)$ observed on samples with high particle contents (e.g. A_10) is due to additional electrical charge conduction brought by the particles. This will be discussed below. At high frequencies, tiny differences in $\tan (\delta)$ are possibly due to modifications in the amorphous phase (different crystallinity ratios, broader alpha transition...).
The effect of the dispersion state on dielectric properties of the materials is discussed now. Since $\varepsilon^{\prime}(\omega)$ can be affected by dielectric losses at low frequencies, the permittivities at $50 \mathrm{~Hz}$ are considered. They are plotted as a function of the BaTiO3 amount in Figure 4 for both sets of dispersion states $\mathrm{A}$ and $\mathrm{S}$. The dielectric loss factors at $0.1 \mathrm{~Hz}$ are plotted in Figure 5.

The data in Figure 4 reflect the increase of $\varepsilon_{\mathrm{r}}$ ' as the $\mathrm{BaTiO} 3$ amount increases, as already mentioned. It shows that $\varepsilon_{\mathrm{r}}$ ' is slightly influenced by the dispersion state. Indeed, samples with aggregated particles (set A) have a higher $\varepsilon_{\mathrm{r}}$ ' than those with well dispersed nanoparticles (set $\mathrm{S}$ ). These results may be explained by polarization couplings between close-by particles with high- $\varepsilon$ within an aggregate [12]. As a consequence of these interactions, an aggregate may polarize like particles with similar permittivity as the elementary particles and overall diameter of the aggregate. As aggregates contain both particles and polymer, the effective volume fraction they occupy is higher than the particle volume fraction, which overall results in a higher effective permittivity of the composite. However, this increase of the composite permittivity is related to higher local electric field

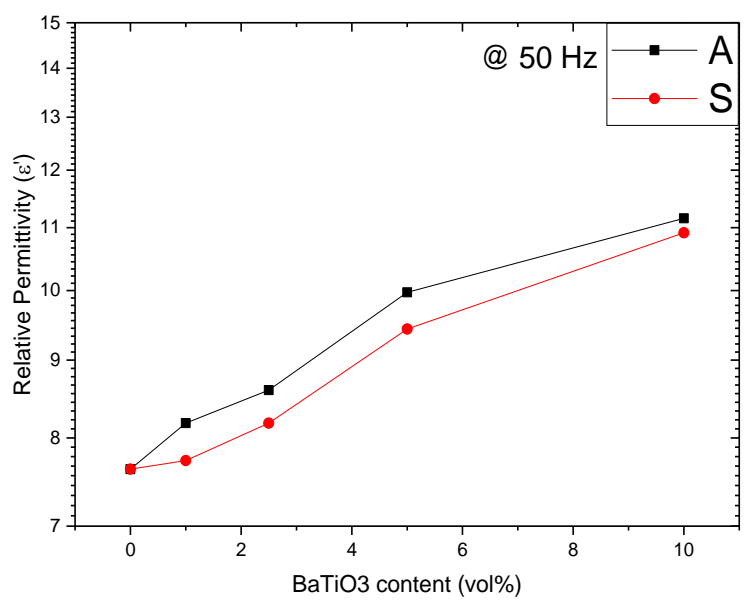

Figure 4: Effective permittivity at $50 \mathrm{~Hz}$ for both well dispersed and aggregated sample as a function of the $\mathrm{BaTiO} 3$ volume content.

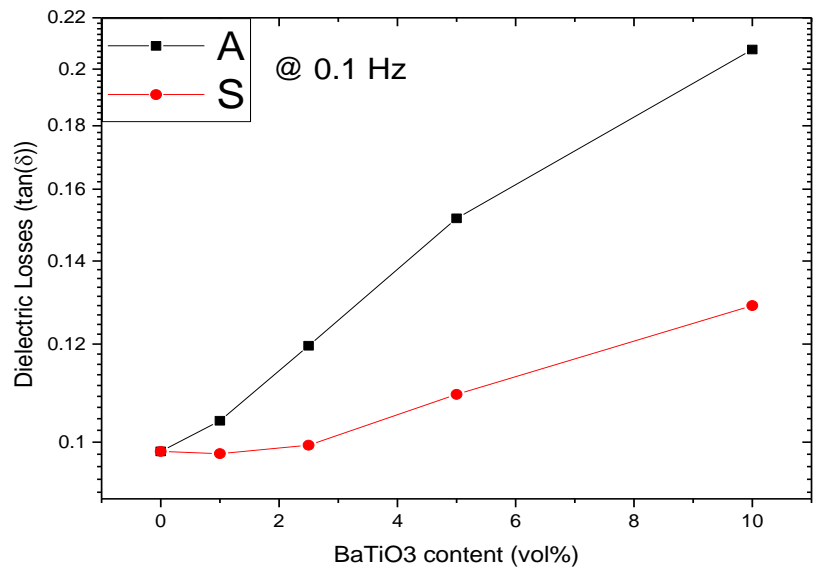

Figure 5: Dielectric losses $\tan (\delta)$ at $0.1 \mathrm{~Hz}$ for both well dispersed and aggregated sample as a function of the $\mathrm{BaTiO}_{3}$ volume content. 
enhancement in the polymer matrix, which may be detrimental for the electrical breakdown strength [13].

It is well known that the nanoparticle dispersion state strongly influences the electrical charge transfer in composites [15]. The difference of dielectric losses induced by adding $\mathrm{BaTiO}_{3}$ nanoparticles with various dispersion states is illustrated in Figure 5. The increase of $\tan (\delta)$ at $0.1 \mathrm{~Hz}$ is observed for both sets of $\mathrm{A}$ and $\mathrm{S}$ samples. As explained above, this is due to the higher conductivity of either $\mathrm{BaTiO}_{3}$ particles themselves or $\mathrm{BaTiO}_{3} / \mathrm{PVdF}-\mathrm{HFP}$ interfaces. Figure 5 also shows that the increase of $\tan (\delta)$ is higher for the samples with aggregated particles (set A). This might be explained by local percolation of aggregated particles, which would increase the conductivity and thus dielectric losses [16]. This local conductivity would also induce a capacitive contribution by the associated MWS polarization of the particles or aggregates, which would increase the permittivity $\varepsilon^{\prime}(\omega)$ to a larger extent in samples with aggregated particles, as observed in Figure 3. As a result, using model systems with different, controlled dispersion states and/or aggregate structures may enable probing electrical charge transfer between particles and thus studying electrical conduction mechanisms in interphases.

Full analysis of dielectric measurements conducted at different temperatures will give access to the quantitative evolution of the conductivity and permittivity and of the dielectric losses associated to interfacial MWS polarization. Moreover, the dielectric behavior at low and high electric field will be investigated by measuring D-E hysteresis loops.

\section{CONCLUSION}

In this work, a method of nanoparticle dispersion has been developed to elaborate composite materials with controlled dispersion states. Dielectric characterization at low field has shown that particle aggregation has a double effect on the dielectric properties of nanocomposites materials. First, it enhances the effective permittivity by a constant factor over the whole range of frequency, which was attributed to polarization coupling between particles. Secondly, the increased loss factor observed at low frequency $(\omega<1-10 \mathrm{~Hz})$ was attributed to interfacial MWS polarization of the particles.

We have seen that different particles aggregation states in nanocomposites reveal inter-particle conduction mechanisms via percolation. This relation may be used to electrically characterize the polymer/particles interphases by comparing at both very controlled aggregated and well dispersed states, the effect of particle surface chemistry (i.e. different interfacial features) onto the dielectric properties of the nanocomposites.

\section{ACKNOWLEDGMENT}

The authors thank Alessio Marani, Francesco Pedroli, Baptiste Gros, Olivier Sanseau and Mathieu Tauban for fruitful scientific discussions.

\section{REFERENCES}

[1] H. Luo et al., "Interface design for high energy density polymer nanocomposites," Chem. Soc. Rev., vol. 48, no. 16, pp. 4424-4465, Sep. 2019, doi: 10.1039/C9CS00043G.

[2] M. Guo, J. Jiang, Z. Shen, Y. Lin, C.-W. Nan, and Y. Shen, "HighEnergy-Density Ferroelectric Polymer Nanocomposites for Capacitive Energy Storage: Enhanced Breakdown Strength and Improved Discharge Efficiency,” Materials Today, vol. 29, pp. 49-67, Oct. 2019, doi: 10.1016/j.mattod.2019.04.015.

[3] T. J. Lewis, "Interfaces: nanometric dielectrics," Journal of Physics D: Applied Physics, vol. 38, no. 2, pp. 202-212, Jan. 2005, doi: 10.1088/0022-3727/38/2/004.

[4] X. Zhang et al., "Superior Energy Storage Performances of Polymer Nanocomposites via Modification of Filler/Polymer Interfaces," Advanced Materials Interfaces, vol. 5, no. 11, p. 1800096, Jun. 2018, doi: 10.1002/admi.201800096.

[5] L. Chen, T. D. Huan, and R. Ramprasad, "Electronic Structure of Polyethylene: Role of Chemical, Morphological and Interfacial Complexity," Scientific Reports, vol. 7, no. 1, Dec. 2017, doi: 10.1038/s41598-017-06357-y.

[6] E. Kubyshkina and M. Unge, "Impact of interfacial structure on the charge dynamics in nanocomposite dielectrics," Journal of Applied Physics, vol. 125, no. 4, p. 045109, Jan. 2019, doi: 10.1063/1.5078800.

[7] K. Wu et al., "The effects of nanoparticles and organic additives with controlled dispersion on dielectric properties of polymers: Charge trapping and impact excitation," Journal of Applied Physics, vol. 120, no. 5, p. 055102, Aug. 2016, doi: 10.1063/1.4959771.

[8] E. Kubyshkina, "Ab initio modelling of interfaces in nanocomposites for high voltage insulation."

[9] G. Teyssedre and C. Laurent, "Advances in high-field insulating polymeric materials over the past 50 years," IEEE Electr. Insul. Mag., vol. 29, no. 5, pp. 26-36, Sep. 2013, doi: 10.1109/MEI.2013.6585854.

[10] J. Su and J. Zhang, "Recent development on modification of synthesized barium titanate (BaTiO3) and polymer/BaTiO3 dielectric composites," Journal of Materials Science: Materials in Electronics, vol. 30, no. 3, pp. 1957-1975, Feb. 2019, doi: 10.1007/s10854-0180494-y.

[11] M. Mackey et al., "Reduction of Dielectric Hysteresis in Multilayered Films via Nanoconfinement," Macromolecules, vol. 45, no. 4, pp. 19541962, Feb. 2012, doi: 10.1021/ma202267r.

[12] X. Huang, B. Sun, Y. Zhu, S. Li, and P. Jiang, "High-k polymer nanocomposites with 1D filler for dielectric and energy storage applications," Progress in Materials Science, vol. 100, pp. 187-225, Feb. 2019, doi: 10.1016/j.pmatsci.2018.10.003.

[13] J. I. Roscow, C. R. Bowen, and D. P. Almond, "Breakdown in the Case for Materials with Giant Permittivity?," ACS Energy Lett., vol. 2, no. 10, pp. 2264-2269, Oct. 2017, doi: 10.1021/acsenergylett.7b00798.

[14] X. Hu, K. Yi, J. Liu, and B. Chu, "High Energy Density Dielectrics Based on PVDF-Based Polymers," Energy Technology, vol. 6, no. 5, pp. 849-864, 2018, doi: 10.1002/ente.201700901.

[15] Z.-H. Shen et al., "Space charge effects on the dielectric response of polymer nanocomposites," Applied Physics Letters, vol. 111, no. 9, p. 092901, Aug. 2017, doi: 10.1063/1.4991079.

[16] G. Zhang et al., "Interfacial Polarization-Induced Loss Mechanisms in Polypropylene/BaTiO3 Nanocomposite Dielectrics," Chem. Mater., vol. 28, no. 13 , pp. 4646-4660, Jul. 2016, doi: 10.1021/acs.chemmater.6b01383. 